\title{
Simultaneous characterization of rotational and translational diffusion of optically anisotropic particles by optical microscopy
}

\author{
Fabio Giavazzi ${ }^{1}$, Catalina Haro-Pérez ${ }^{2}$ and Roberto Cerbino ${ }^{1}$ \\ 1 Dipartimento di Biotecnologie Mediche e Medicina Traslazionale, Università degli Studi di \\ Milano, via F.lli Cervi 93, 20090 Segrate, Italy \\ 2 Departamento de Ciencias Básicas, Universidad Autónoma Metropolitana-Azcapotzalco, Av. \\ San Pablo 180, 02200 México D.F., Mexico \\ E-mail: fabio.giavazzi@unimi.it
}

\begin{abstract}
We probe the roto-translational Brownian motion of optically anisotropic particles suspended in water with a simple and straightforward optical microscopy experiment that does not require positional or rotational particle tracking. We acquire a movie of the suspension placed between two polarizing elements and we extract the translational diffusion coefficient $D_{T}$ and the rotational diffusion coefficient $D_{R}$ from the analysis of the temporal correlation properties of the spatial Fourier modes of the intensity fluctuations in the movie. Our method is successfully tested with a dilute suspension of birefringent spherical colloidal particles obtained by polymerizing an emulsion of droplets of liquid crystal in a nematic phase, whose roto-translational dynamics is found to be well described by theory. The simplicity of our approach makes our method a viable alternative to particle tracking and depolarized dynamic light scattering.
\end{abstract}

Submitted to: J. Phys.: Condens. Matter 


\section{Introduction}

Dispersions of colloidal particles in a liquid are among the most studied soft condensed matter systems for both fundamental science and industrial applications [1]. The dynamics of these dispersions has been traditionally studied with a wide portfolio of methods that include Video Particle Tracking (VPT), Dynamic Light Scattering (DLS), and Fluorescence Correlation Spectroscopy (FCS) $[2,3]$. All these methods enable characterizing the translational dynamics of the particles and, when the particles are non-spherical or optically anisotropic, also their rotational ones. In particular, Depolarized Dynamic Light Scattering (DDLS) is the extension of DLS that relies on the fact that anisotropic particles alter the state of polarization of light that impinges on them $[2,4,5]$ : if the dispersion is placed between cross-polarizers, fluctuations in the scattering intensity are observed not only when the particles translate but also when they rotate. The study of these intensity fluctuations enable accessing both the translational and the rotational diffusion coefficients of the particles. These two quantities can be used to size the colloidal particles or, if their size is known, to probe the viscoelastic properties of the hosting medium, the so called microrheology $[6,7]$. This application of Brownian motion as probe of the translational and rotational degrees of freedom appears particularly intriguing in view of the translational-rotational decoupling that occurs in several systems ranging from colloidal crystals, glasses and gels [8-10] to supercooled fluids with dynamic heterogeneity [11, 12].

Recently, a novel technique named Differential Dynamic Microscopy (DDM) was proposed for the quantification of the translational diffusion coefficient of arbitrarily small colloidal particles $[13,14]$. DDM is based on using a commercial optical microscope equipped with a camera instead of a more expensive and dedicated DLS laser setup. Being based on the time analysis of spatial Fourier transforms of real-space images, DDM opens to a space-resolved investigation of the sample dynamics with obvious advantages for spatially heterogenous systems [15]. Extending DDM to the measurement of rotational diffusion would be extremely important for both sizing and microrheology applications since rotational diffusion depends cubically on the particle size, in contrast with the linear dependence exhibited by translational diffusion. This stronger dependence implies a larger sensitivity to particle size in sizing applications and to the forces exerted by the medium on the particles in microrheological ones.

Here we demonstrate that polarized-DDM (p-DDM), basically DDM with the addition of two polarizing elements [16], enables a very simple and accurate characterization of the translational and rotational diffusion of optically anisotropic particles dispersed in water. We provide a self-contained theoretical description of the problem and we discuss in detail the optical conditions under which pDDM can be applied and provides meaningful results. Finally, we discuss the connection of p-DDM with related methods aimed to characterize rotational diffusion of colloidal particles [17-19].

\section{Theory}

In this Section we will describe the theoretical framework needed to plan, execute and interpret microscopy experiments with optically anisotropic particles for the extraction of quantitative information on their roto-translational dynamics. Our analysis is based on three main pillars: (i) a recent extension by Degiorgio et al. [20] of the scattering theory from optically anisotropic particles [2]; (ii) a modification of the analysis in Ref. [14] to include polarization effects in the description of DDM experiments; (iii) the identification of a suitable experimental geometry for p-DDM experiments. 
Scattering from optically anisotropic uniaxial particles

An optically anisotropic uniaxial particle is described by a polarizability tensor that admits a diagonal form with elements $\alpha_{1}, \alpha_{2}$ and $\alpha_{3}$ with $\alpha_{2}=\alpha_{1}$. The anisotropy parameter is defined as $\beta=\alpha_{3}-\alpha_{1}$. If such particle is dispersed in a solvent of refractive index $n_{s}$ then its average (excess) polarizability is $\alpha=\frac{2 \alpha_{1}+\alpha_{3}}{3}-V\left(n_{s}^{2}-1\right)$, where $V$ is the particle volume. When illuminated by a plane wave $\mathbf{A}_{\mathbf{0}}(z)=\mathbf{E}_{\mathbf{0}} e^{-j k z}$ of wave-number $k$, this particle emits a scattered field that, in addition to the component $E_{V V}$ that is parallel to $\mathbf{E}_{\mathbf{0}}$, also bears a perpendicular component $E_{V H}$. Following Van de Hulst [21], one has:

$$
E_{V V}=S_{V V} \frac{e^{-j k r+j k z}}{j k r} A_{0}(z)
$$

and

$$
E_{V H}=S_{V H} \frac{e^{-j k r+j k z}}{j k r} A_{0}(z)
$$

where the dimensionless amplitudes $S_{V V}$ and $S_{V H}$ are functions of the scattering angle $\theta_{s}$ measured with respect to the incident direction $z$, and depend upon the orientation of the particle.

According to standard treatment [2], in the Rayleigh-Gans approximation (weakly-scattering particles) and for small scattering angles, the scattering amplitudes $S_{V V}$ and $S_{V H}$ are given by the following expressions:

$$
\begin{aligned}
& S_{V V}=j k^{3} \alpha+j k^{3} \beta \sqrt{\frac{16 \pi}{45}} Y_{2,0}(\theta, \phi) \\
& S_{V H}=j k^{3} \beta \sqrt{\frac{2 \pi}{15}} j\left[Y_{2,-1}(\theta, \phi)+Y_{2,1}(\theta, \phi)\right]
\end{aligned}
$$

that are written in terms of the the spherical harmonic function $Y_{l, m}(\theta, \phi)$ of order $l, m$. We note that both $S_{V V}$ and $S_{V H}$ are purely imaginary quantities, which implies that the scattered field in the forward direction (and in the far -field of the particle) is in quadrature with the transmitted field. An immediate consequence of this fact is that it is not possible to observe interference of the forward scattered light with the transmitted beam. This prevents the so-called heterodyne detection of scattering signal from these particles, unless special experimental precautions are taken. A simple and elegant solution to this problem is the one proposed in Ref.[5], where the authors insert in the collection arm between the sample and the analyzing element a quarter-wave plate having the fast axis parallel to the axis of the polarizer, enabling thereby the interference of transmitted and scattered light.

Very recently, it has been pointed out by Degiorgio et al. in Ref.[20], that Eqs. 3, 4 are not compatible with the energy conservation as expressed by the optical theorem [21], as they do not account for the effect of radiation reaction. The self-consistent treatment in Ref.[20] leads to the following modified expressions, accurate up to the second order in $k^{3} \alpha$ :

$$
\begin{aligned}
& S_{V V}=\left(j k^{3} \alpha+\frac{2}{3} k^{6} \alpha^{2}+\frac{4}{27} k^{6} \beta^{2}\right)+\left[j k^{3} \beta+\frac{2}{9} k^{6}\left(\alpha_{3}^{2}-\alpha_{1}^{2}\right)\right] \sqrt{\frac{16 \pi}{45}} Y_{2,0}(\theta, \phi) \\
& S_{V H}=\left[j k^{3} \beta+\frac{1}{3} k^{6}\left(\alpha_{3}^{2}-\alpha_{1}^{2}\right)\right] \sqrt{\frac{2 \pi}{15}} j\left[Y_{2,-1}(\theta, \phi)+Y_{2,1}(\theta, \phi)\right]
\end{aligned}
$$


Compared to Eqs. 3 and 4, new terms arise for both $S_{V V}$ and $S_{V H}$. These new terms are purely real and ensure the existence of a component of the scattered field that is in phase with the transmitted field. Whether the amplitude of such component becomes measurable in practice in experiments depends on the mismatch between the average polarizability of the particle and the polarizability of the solvent, as expressed by Eqs. 5 and 6 [22]. The result of Degiorgio et al. [20] is relevant for the experiments described here, since in this configuration DDM is in fact a heterodyne scattering technique [15, 23]. Our description will thus make use of the more accurate expressions for $S_{V V}$ and $S_{V H}$ given by Eqs. 5 and 6 .

\section{Microscopy of optically anisotropic uniaxial particles: real space}

When a collection of such particles is observed with a microscope, the image formation process can be described within the general framework of Ref. [14], where the image formation is treated as a linear space-invariant process. Within this model, the coherence properties of the light source, the details of the collection optics and the intrinsic scattering properties of the sample determine an effective three-dimensional (3D) point-spread function (PSF) $K(\mathbf{x}, z)$. The microscope image $I(\mathbf{x})$ can be thus obtained as a weighted sum of all the contributions that are originated by the different layers of the sample, each layer being described by a transmittance function $t(\mathbf{x}, z) \equiv \frac{E(\mathbf{x}, z+d z)}{A_{0}(z)}$. Here, as above, $A_{0}(z)$ is the incident field that impinges on the layer and $E(\mathbf{x}, z+d z)$ is the total field (scattered + transmitted) emerging from the layer.

Optically isotropic particles In Ref. [14], this summation was performed for a collection of identical particles described by the same scattering amplitude $a=a_{A}+j a_{P}$. Under the assumption of a weakly scattering sample, the relation $t(\mathbf{x}, z)=1+a \cdot c(\mathbf{x}, z) d z / k^{2}$ holds, where

$$
c(\mathbf{x}, z)=\sum_{n} \delta\left(\mathbf{x}-\mathbf{x}_{n}\right) \delta\left(z-z_{n}\right)
$$

is the number density of particles and $d z$ is the layer thickness. The resulting expression for the normalized intensity distribution reads

$$
s(\mathbf{x}, t)=\frac{I-I_{0}}{I_{0}}=\iiint d \mathbf{x}^{\prime} d z^{\prime} K\left(\mathbf{x}-\mathbf{x}^{\prime},-z^{\prime}\right) c\left(\mathbf{x}^{\prime}, z^{\prime}, t\right)
$$

where $I_{0}$ is the average intensity in the image and where we have considered the time-dependence in the particles positions. In Eq. 8, the scattering amplitude $a$ is incorporated in the effective PSF that can be written as $K=a_{A} T_{A F}+j a_{P} T_{P F}$, where $T_{A F}$ and $T_{P F}$ are the PSF for amplitude and phase objects, respectively [14]. Phase objects are those for which scattering is in quadrature, whereas for amplitude objects scattering is in phase with the transmitted field. In general, an optically isotropic particle acts in a mixed way, altering both the amplitude and the phase of light [21]. This suggests to rewrite Eq. 8 as

$$
\begin{array}{r}
s(\mathbf{x}, t) \quad=\iiint d \mathbf{x}^{\prime} d z^{\prime} T_{A F}\left(\mathbf{x}-\mathbf{x}^{\prime},-z^{\prime}\right) c_{A}\left(\mathbf{x}^{\prime}, z^{\prime}, t\right)+ \\
+j \iiint d \mathbf{x}^{\prime} d z^{\prime} T_{P F}\left(\mathbf{x}-\mathbf{x}^{\prime},-z^{\prime}\right) c_{P}\left(\mathbf{x}^{\prime}, z^{\prime}, t\right)
\end{array}
$$

where $c_{A}=a_{A} c$ and $c_{P}=a_{P} c$ can be thought of as densities associated to the scattering amplitude of particles that scatter in phase and in quadrature with the transmitted beam, respectively. Being the scattering amplitude $a$ identical for all particles, the above-reported equations are not immediately applicable as it is to optically anisotropic particles, unless they are all oriented along the same direction. 


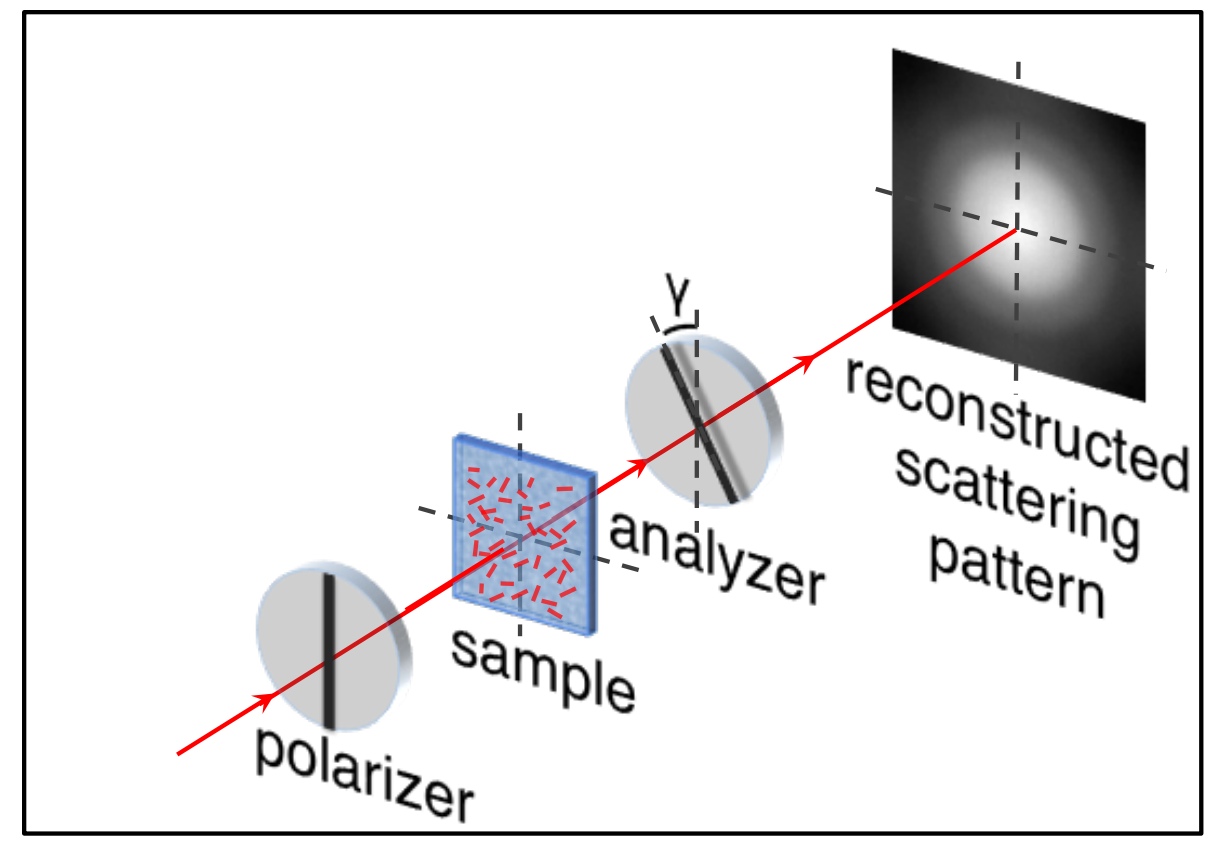

Figure 1. Sketch of the experimental setup used for p-DDM experiments. Unpolarized light emitted by a source (not shown) is polarized by using a polarized element oriented in the vertical (V) direction. Vertically polarized light interacts with the suspension, in which the optical axis (represented as a red line) of each particle is randomly oriented. As a consequence, the light emerging from the sample also bears a horizontally polarized component. A second polarizing element (analyser) oriented at an angle $\gamma$ with respect to the vertical direction is used to filter the light scattered by the sample. A time-lapse acquisition of images in this configuration is acquired and used to perform an imaging-based depolarized dynamic light scattering experiment (see text for details).

Optically anisotropic particles However, this difficulty can easily be bypassed by defining the densities $c_{A}$ and $c_{P}$ in a way that accounts for the possible different orientation of each particle with respect to the incident polarization. We consider for definiteness the experimental setup sketched in Fig. 1, where the illumination beam is polarized along the vertical direction and the light transmitted and scattered by the sample passes through an analyzer rotated by an angle $\gamma$ with respect to the vertical direction. In these conditions the transmittance function for a sample layer of thickness $d z$ is defined as $t(\mathbf{x}, z) \equiv \frac{E(\mathbf{x}, z+d z)}{A_{0}(z) \cos \gamma}$. Here $E(\mathbf{x}, z+d z)$ is now the projection along the analyzer of the total field (scattered + transmitted) emerging from the layer, and $\cos \gamma$ is a geometrical correction that ensures $t(\mathbf{x}, z)=1$ if no scatterers are present within the layer. Use of Eqs. 1 and 2 leads thus again to Eq.9 where now

$$
\begin{aligned}
& c_{A}(\mathbf{x}, z, t)=\Re\left[c_{V V}(\mathbf{x}, z, t)+c_{V H}(\mathbf{x}, z, t) \tan \gamma\right] \\
& c_{P}(\mathbf{x}, z, t)=\Im\left[c_{V V}(\mathbf{x}, z, t)+c_{V H}(\mathbf{x}, z, t) \tan \gamma\right]
\end{aligned}
$$

and where the newly introduced quantities

$$
c_{V V}(\mathbf{x}, z, t)=\sum_{n} S_{V V}\left(\theta_{n}(t), \phi_{n}(t)\right) \delta\left(\mathbf{x}-\mathbf{x}_{n}(t)\right) \delta\left(z-z_{n}(t)\right)
$$




$$
c_{V H}(\mathbf{x}, z, t)=\sum_{n} S_{V H}\left(\theta_{n}(t), \phi_{n}(t)\right) \delta\left(\mathbf{x}-\mathbf{x}_{n}(t)\right) \delta\left(z-z_{n}(t)\right)
$$

can be thought of as densities associated to the scattering amplitude of polarized and depolarized scattering. It is interesting to note that the case of optically isotropic particles can be obtained as a special case with $S_{V V}=\left(j k^{3} \alpha+\frac{2}{3} k^{6} \alpha^{2}\right)$ and $S_{V H}=0$. This leads to $c_{A}=\frac{2}{3} k^{6} \alpha^{2} c$ and $c_{P}=k^{3} \alpha c$, which agrees with the analysis in Ref. [14], provided that $a_{A}=\frac{2}{3} k^{6} \alpha^{2}$ and $a_{P}=k^{3} \alpha$.

\section{Microscopy of optically anisotropic uniaxial particles: Fourier space}

The two-dimensional $(2 D)$ Fourier transform $\hat{s}(\mathbf{q}, t)=\int s(\mathbf{x}, t) e^{-j \mathbf{q} \cdot \mathbf{x}} d \mathbf{x}$ of the normalized intensity distribution in Eq. 9 reads

$$
\hat{s}(\mathbf{q}, t)=\int d q_{z} \tilde{T}_{A F}\left(\mathbf{q}, q_{z}\right) \tilde{c}_{A}\left(\mathbf{q}, q_{z}, t\right)+j \int d q_{z} \tilde{T}_{P F}\left(\mathbf{q}, q_{z}\right) \tilde{c}_{P}\left(\mathbf{q}, q_{z}, t\right)
$$

where the symbol $\hat{\square}$ is used from hereon to indicate the $2 \mathrm{D}$ Fourier transform of the corresponding quantity and the symbol $\tilde{\square}$ is used for the 3D Fourier transform. Starting from Eq. 14 it is possible to calculate the (non-normalized) image intermediate scattering function $F_{i}(\mathbf{q}, \Delta t)=\left\langle\hat{s}^{*}(q, 0) \hat{s}(q, \Delta t)\right\rangle[15]$. A relatively simple expression for $F_{i}(\mathbf{q}, \Delta t)$ can be obtained as shown in Appendix for a dilute suspension of non-interacting spherical particles of radius $R$ in the small-q limit, where the axial dynamics does not substantially affect the image correlation function $[14,24]$ and one has

$$
F_{i}(\mathbf{q}, \Delta t) \simeq\left[A_{1}^{\prime}(\mathbf{q}) e^{-D_{T} q^{2} \Delta t}+A_{2}^{\prime}(\mathbf{q}) e^{-\left(D_{T} q^{2}+6 D_{R}\right) \Delta t}\right]
$$

where detailed expression for the amplitudes $A_{1 / 2}^{\prime}(q)$ are provided in Appendix. In Eq. 15, the

coefficients $D_{R}=\frac{k_{B} T}{8 \pi \eta R^{3}}$ and $D_{T}=\frac{k_{B} T}{6 \pi \eta R}$ are the the Stokes-Einstein predictions for the rotational and translational diffusion coefficient, respectively, where $k_{B}$ is the Boltzmann constant, $T$ the absolute temperature and $\eta$ the viscosity of the solvent.

The image intermediate scattering function in Eq. 15 can be also written in its normalized version

$$
f_{i}(\mathbf{q}, \Delta t)=\frac{F_{i}(\mathbf{q}, \Delta t)}{F_{i}(\mathbf{q}, 0)}=f_{1}(\mathbf{q}) e^{-\Gamma_{1}(q) \Delta t}+f_{2}(\mathbf{q}) e^{-\Gamma_{2}(q) \Delta t}
$$

where we have defined $\Gamma_{1}(q)=D_{T} q^{2}+6 D_{R}, \Gamma_{2}(q)=D_{T} q^{2}, f_{1}(\mathbf{q})=A_{1}^{\prime}(\mathbf{q}) /\left(A_{1}^{\prime}(\mathbf{q})+A_{2}^{\prime}(\mathbf{q})\right)$ and $f_{2}(\mathbf{q})=A_{2}^{\prime}(\mathbf{q}) /\left(A_{1}^{\prime}(\mathbf{q})+A_{2}^{\prime}(\mathbf{q})\right)$, with $f_{1}+f_{2}=1$. The dynamics of the suspension is thus described by two modes with relative amplitude $f_{1}$ and $f_{2}$ and different rates. We note that $f_{i}$ has exactly the same form of the intermediate scattering function which is expected for a heterodyne DDLS experiment [2] and, similarly to DDLS, the q-resolved study of the two modes allows the simultaneous determination of both $D_{T}$ and $D_{R}$.

\section{Materials and methods}

\subsection{Sample synthesis and preparation}

The sample is an aqueous suspension of spherical particles with a polymerized nematic liquid crystal core [19, 25-27]. The optical birefringence typical of the nematic phase makes the particles optically anisotropic with a uniaxial symmetry. The particles used in this study have been prepared 
according to the protocol described in Ref.[25] by emulsification of the liquid crystal (RM257, Merck) in water at a temperature of $80^{\circ} \mathrm{C}$, in which the phase is nematic. With a UV lamp the photoinitiator Darocur 1173 (Ciba), initially added to the solution, is activated and allows the RM257 to polymerize in order to create droplets with a nematic core where the liquid crystal is confined in an axial configuration. After this procedure, the particles are consistently polydisperse. The degree of polydispersity is then reduced by using a method that makes use of iterated liquid-solid colloidal phase transitions induced by attractive depletion interactions [28]. After this procedure the final mass fraction of the dispersion is $(2.4 \pm 0.1) \times 10^{-2}$. To determine the particle hydrodynamic radius we dilute the sample to a final mass fraction $(1.1 \pm 0.1) \times 10^{-5}$, by adding deionized water of resistivity $20 \mathrm{M} \Omega \mathrm{cm}$. The dilute suspension is loaded into a capillary tube (Vitrocom Inc.) with rectangular section and thickness along the optical axis equal to $50 \mu \mathrm{m}$. The sample temperature is kept at $20.0 \pm 0.1{ }^{\circ} \mathrm{C}$ for the whole duration of the experiments by means of a commercial microscope hot stage (Instec HCS302). At this temperature, the viscosity of the solvent is $\eta=1.02 \pm 0.02 \mathrm{mPa}$ $\stackrel{\mathrm{u}}{\mathrm{s}}$. A first characterization of the particles is obtained with particle tracking performed on bright field movies (100 fps) that provides an average hydrodynamic radius of $191 \pm 7 \mathrm{~nm}$. On the same sample we also performed p-DDM measurements, as described in the following.

\subsection{Polarized differential dynamic microscopy ( $p$-DDM)}

The particles in suspension are imaged by using a Nikon Eclipse Ti-E commercial microscope equipped with a Hamamatsu Orca Flash 4.0 v2 camera. We used a microscope objective with magnification $M=20 X$ and numerical aperture $N A=0.4$. The pixel size after magnification and $2 \times 2$ binning is equal to $0.645 \mu \mathrm{m}$. With respect to standard DDM, where no polarizing elements are used, here we sandwich the sample between two polarizers that are mutually oriented at an angle $\gamma=75^{\circ}$ (p-DDM configuration). A sequence of $N=100000$ square images $I(\mathbf{x}, t)$ is acquired with a frame rate $r=800$ fps. Here $\mathbf{x}=(x, y)$, the $z$ axis is oriented along the microscope optical axis and the time variable can assume only discrete values that are integer multiples of $1 / r$. Each image is obtained with exposure time $t_{\text {exp }}=1.2 \mathrm{~ms}$ and has $N_{\text {pix }}=128$ pixels per side.

A representative image acquired in the described condition is shown in Fig. 2a. For comparison, in Fig. 2b a bright-field image of the same sample is also reported. As it can be appreciated also from the supplementary movie M1, while the translational dynamics of the particles is well captured in both configuration, only in the polarized set-up the rotational dynamics produces a clear signal, in the form of an intermitted blinking of the intensity associated to each particle. Once acquired and saved on disk, an image sequence is analyzed as in standard DDM. For each time delay $\Delta t=k / r$ of interest, with $k=1, \ldots, N-1$ the difference signal $d\left(\mathbf{x}, t_{0}, \Delta t\right)=[I(\mathbf{x}, t+\Delta t)-I(\mathbf{x}, t)] / I_{0}$ is

calculated and its spatial Fourier power spectrum is computed by using a Fast Fourier Transform (FFT) routine. In the presence of stationary or quasi-stationary statistical processes, an average over power spectra with the same $\Delta t$ but different reference time $t_{0}$ is obtained, which increases the statistical accuracy of the data. This leads to the so called image structure function (ISF)

$$
d_{i}(\mathbf{q}, \Delta t)=\left\langle\left|F F T\left[D\left(\mathbf{x}, t_{0}, \Delta t\right)\right]\right|^{2}\right\rangle_{t_{0}}
$$

that captures the dynamics of the sample as a function of the two-dimensional scattering wavevector $\mathbf{q}$ and of the delay time $\Delta t$. The ISF is connected to the (normalized) intermediate scattering function $f_{i}(\mathbf{q}, \Delta t)$ [2] by the relation

$$
d_{i}(\mathbf{q}, \Delta t)=2 A^{\prime}(\mathbf{q})\left[1-f_{i}(\mathbf{q}, \Delta t)\right]+2 B(\mathbf{q})
$$



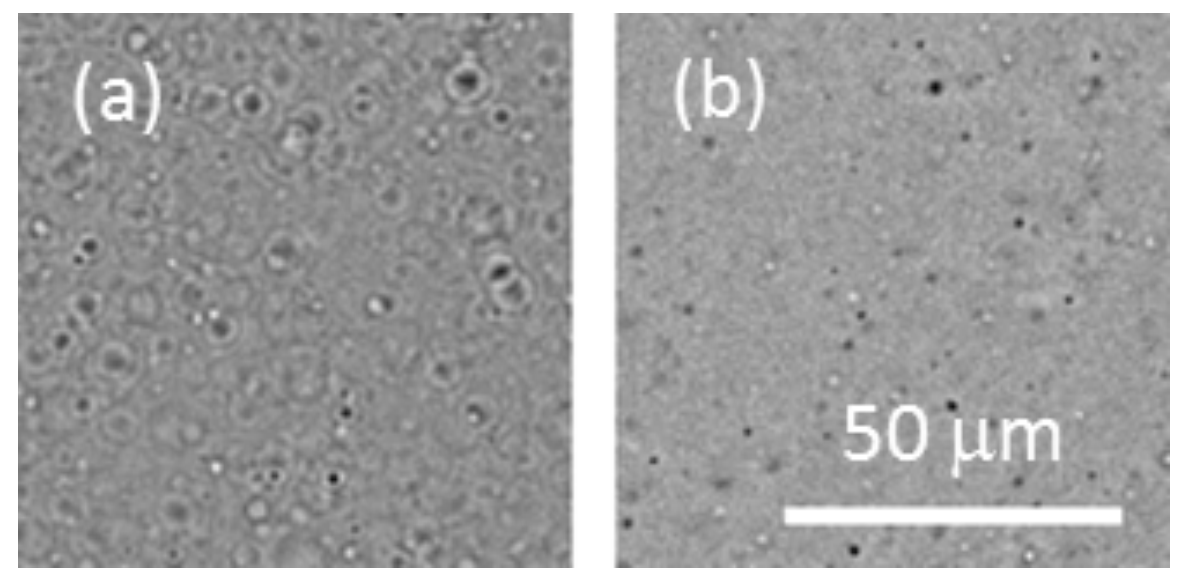

Figure 2. (a) Bright-field image of a suspension of optically anisotropic particles. (b) Image of the same sample observed between polarizers mutually oriented at $75^{\circ}$. The scale bar corresponds to $50 \mu \mathrm{m}$. In both cases a background image, obtained as the average of a stack of 100000 images, was subtracted to eliminate static contributions. See also Supplementary Movie M1 for a dynamic comparison of the two imaging conditions.

where $B(\mathbf{q})$ is a term that accounts for the camera noise and $A^{\prime}(\mathbf{q})$ is an amplitude term that contains information about the static scattering from the sample and details about the imaging system [15]. As described in Section 2, the intermediate scattering function is given by Eq. 16 and $A^{\prime}(\mathbf{q})=A_{1}^{\prime}(\mathbf{q})+A_{2}^{\prime}(\mathbf{q})$. Here, due to the random orientation of the particles with respect to the polarizing elements, the ISF $d_{i}(\mathbf{q}, \Delta t)$ bears a circular symmetry and we can perform azimuthal averaging of the data to increase the statistical accuracy at each $q=\sqrt{q_{x}^{2}+q_{y}^{2}}$, as previously done in other cases [13, 14, 29-33]. We note however that this is not a general rule and the two-dimensional nature of the ISF provides in general a powerful way to inspect the dynamics along directions in the $\mathbf{q}$ plane that are of particular interest for the problem under study [16].

\section{Results and discussion}

We report in Fig. 3 the intermediate scattering functions obtained with p-DDM for some representative values of the scattering wave-vector $q$. As expected from Eq. 16, the observed relaxation exhibits two different decays for all values of $q$. In principle, fitting of these curves to Eq. 16 provides estimates for $\Gamma_{1}(q)=D_{T} q^{2}+6 D_{R}, \Gamma_{2}(q)=D_{T} q^{2}$, and for the two amplitudes $f_{j}(q)$, with $j=1,2$. However, we find that the dynamics is not fully captured by a sum of two simple-exponential decays, as it would be expected for the roto-translational diffusion of a collection of identical particles. In the presence of a moderate size polydispersity, as in our case, it is usual to replace the single-exponential decay $e^{-\Gamma \Delta t}$ with the expansion $e^{-\bar{\Gamma} \Delta t}\left[1+\frac{\mu_{2}}{2 !}(\Delta t)^{2}-\frac{\mu_{3}}{3 !}(\Delta t)^{3}+\ldots\right]$, where $\mu_{n}=\int(\Gamma-\bar{\Gamma})^{n} G(\Gamma) d \Gamma$ are the central moments of the normalized distribution $G(\Gamma)$ of the decay rates [34]. This form for the intermediate scattering function is such that the mean value $\bar{\Gamma}$ of $G(\Gamma)$ provides an estimate for the average decay rate and $\mu_{2} / \bar{\Gamma}^{2}$, the normalized variance of $G(\Gamma)$, provides an estimate for the width of the distriibution and 


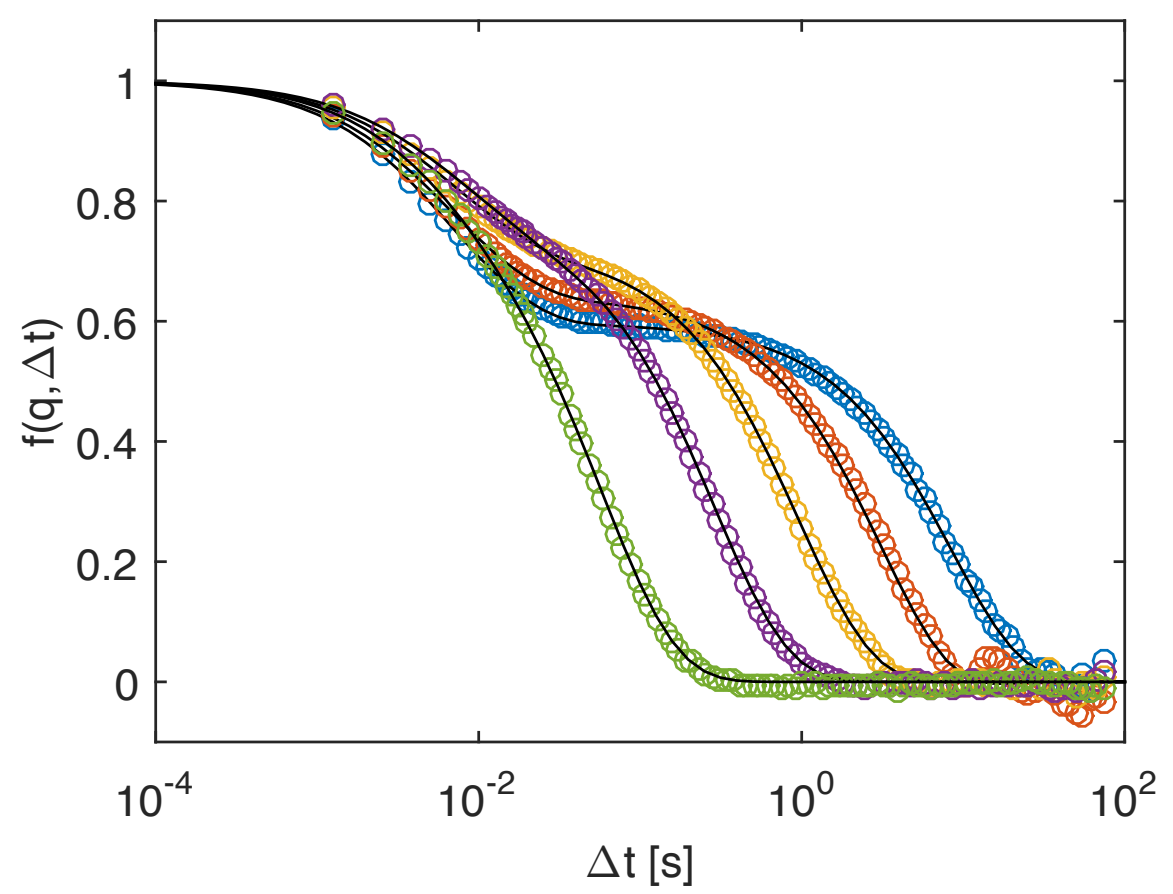

Figure 3. Symbols: intermediate scattering function $f(q, \Delta t)$ obtained in the polarized microscopy experiment for different wave vectors $q=0.38,0.60,1.06,1.81,3.93 \mu^{-1}$. Continuous lines: best fitting curves with the model function given in Eq. 19.

in turn for the sample polydispersity. Using this expansion for both the terms in Eq. 16 we obtain

$$
\begin{aligned}
f_{p o l}(q, \Delta t) & =f_{1} e^{-\Gamma_{1} \Delta t}\left[1+\frac{\mu_{2}^{(1)}}{2 !} \Delta t^{2}-\frac{\mu_{3}^{(1)}}{3 !} \Delta t^{3}\right]+ \\
& +\left(1-f_{1}\right) e^{-\Gamma_{2} \Delta t}\left[1+\frac{\mu_{2}^{(2)}}{2 !} \Delta t^{2}-\frac{\mu_{3}^{(2)}}{3 !} \Delta t^{3}\right],
\end{aligned}
$$

where for notational simplicity we indicate as $\Gamma_{1}$ and $\Gamma_{2}$ the (average) decay rates associated to the two terms. In Fig. 4 we report the results obtained for $\Gamma_{1}(q)$ and $\Gamma_{2}(q)$ with blue squares and orange circles, respectively.

Fitting our data to the theoretical expressions $\Gamma_{1}(q)=D_{T} q^{2}+6 D_{R}$, and $\Gamma_{2}(q)=D_{T} q^{2}$ provides the best estimates $D_{T}=1.14 \pm 0.02 \mu \mathrm{m}^{2} / \mathrm{s}$ and $D_{R}=25.1 \pm 1.0 \mathrm{~s}^{-1}$ for the translational and rotational diffusion coefficients, respectively. By using these values for the diffusion coefficient in the Stokes-Einstein relations for translational and rotational motion, we obtain similar radii, $R=185 \pm 5 \mathrm{~nm}$ and $R=185 \pm 3 \mathrm{~nm}$, respectively. Alternatively, it is possible to use the viscosityand temperature-independent relationship $\frac{D_{T}}{D_{R}}=\frac{4}{3} R^{2}[4]$ to obtain $R=(185 \pm 4) \mathrm{nm}$. All these values are in good agreement with the result $R=191 \pm 7 \mathrm{~nm}$ obtained by VPT .

To probe translational diffusion without contributions from the rotational dynamics, we also performed experiments with bright-field DDM i.e. without any polarizers. For these experiments 


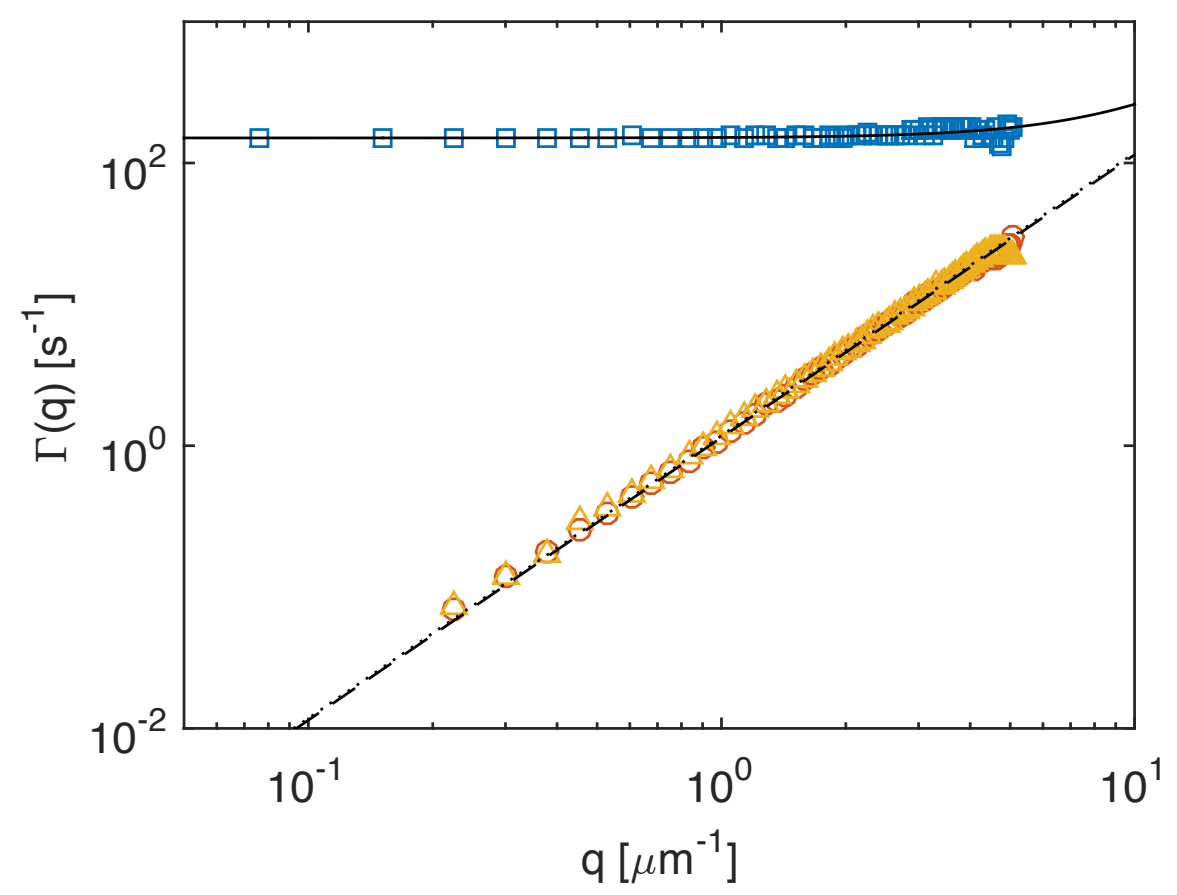

Figure 4. Decorrelation rates obtained from the fit of the structure functions obtained in the polarized (blue squares and orange circles) and bright-field (yellow triangles) microscopy experiments with the model functions given, respectively, in Eq, 19 and 20. Squares and circles represents the decorrelation rates $\Gamma_{1}(q)$ and $\Gamma_{2}(q)$, respectively (see Eq. 19). Triangles represent the decorrelation rate $\Gamma_{3}(q)$ (see Eq.20). The continuous line is the best fit of $\Gamma_{1}(q)$ in the form $6 D_{R}+D_{T} q^{2}$, leading to $D_{R}=25.1 \pm 1.0 \mathrm{~s}^{-1} . D_{T}$ was kept fixed to the value $D_{T}=1.14 \pm 0.02$ $\mu \mathrm{m}^{2} / \mathrm{s}$ obtained from the fit of $\Gamma_{2}(q)$ with a function of the form $D_{T} q^{2}$ (dashed line). The best fit of $\Gamma_{3}$ with the same functional form provides $D_{T}=1.18 \pm 0.02 \mu \mathrm{m}^{2} / \mathrm{s}$ (dotted line).

$N=40000, N_{p i x}=128, t_{e x p}=1.2 \mathrm{~ms}$ and $r=100 \mathrm{fps}$. For all q we find that the intermediate scattering functions are well described by a single-decay, once again well captured by the expansion

$$
f_{b f}(q, \Delta t)=e^{-\Gamma_{3} \Delta t}\left(1+\frac{\mu_{2}^{(3)}}{2 !} \Delta t^{2}-\frac{\mu_{3}^{(3)}}{3 !} \Delta t^{3}\right)
$$

and the extracted decay rate $\Gamma_{3}(q)$ is shown in Fig. 4 (yellow triangles). Fitting of our data to the expression $\Gamma_{3}(q)=D_{T} q^{2}$ gives $D_{T}=1.18 \pm 0.02 \mu \mathrm{m}^{2} / \mathrm{s}$ and $R=179 \pm 5 \mathrm{~nm}$, in agreement with the value extracted with p-DDM and also compatible with the result $R=191 \pm 7 \mathrm{~nm}$ obtained by VPT .

The consistency of our results can be checked by inspecting the polydispersity index $\mu_{2} / \bar{\Gamma}^{2}$, whose q-dependence is shown in Fig. 5 for both p-DDM (blue squares and orange circles) and bright-field DDM (yellow triangles) experiments. As expected, the mean value $0.11 \pm 0.03$ of the polydispersity index associated to roto-translational diffusion (blue squares) is larger than the ones 


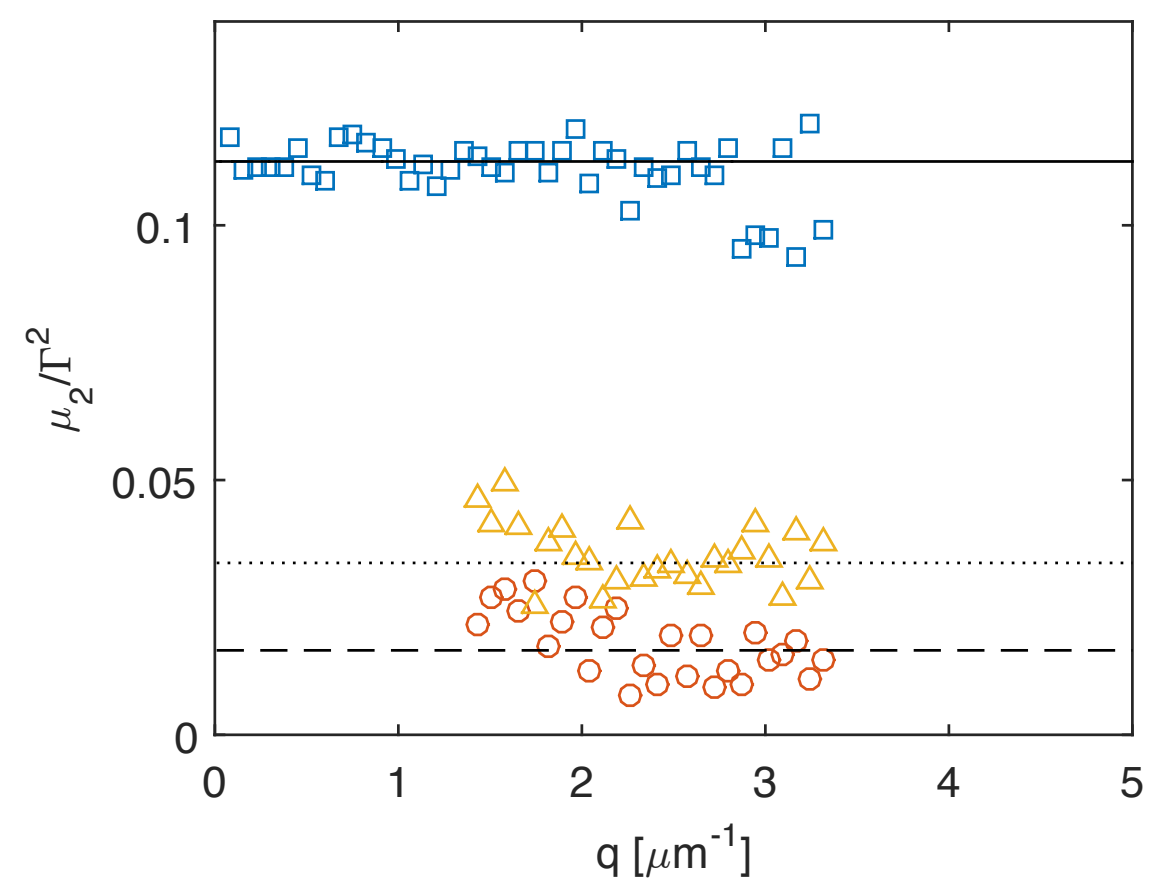

Figure 5. Polydispersity index, defined as the ratio of the second term of the cumulant expansion $\kappa_{2}(q)$ and the square of first cumulant $\Gamma(q)$ of the exponential decays given in 19 (p-DDM) and 20 (bright-field DDM). Blue squares and orange circles are obtained with p-DDM and are associated with the first and the second exponential decay in Eq. 19, respectively; continuous and dashed lines correspond to the respective mean values. Yellow triangles are obtained with bright-field DDM and are associated with the single decay in Eq. 20. The corresponding average value is represented as a dotted line.

extracted from the analysis of translational motion in both p-DDM (orange circles) and brightfield DDM (yellow triangles), which have compatible mean values: $0.02 \pm 0.01$ and $0.035 \pm 0.010$, respectively. In fact, taking into account that in the explored low- $q$ regime: $\Gamma_{1} \propto D_{R} \propto R^{-3}$, while $\Gamma_{2}=\Gamma_{3} \propto D_{T} \propto R^{-1}$, it follows immediately and in agreement with our data that the polydispersity index associated to the rate $\Gamma_{1}$ (i.e. to rotational diffusion in our q-range) should be nine times larger than the ones associated to the rates $\Gamma_{2}$ and $\Gamma_{3}$ (i.e. to translational diffusion).

In our working conditions, we can estimate that the fractional attenuation of the transmitted intensity due to scattering amounts to $t=1.85 \times 10^{-5}$, as estimated from the integral of the scattering intensity. We also performed measurements in the concentration interval $5 \times 10^{-6}<\phi<$ $5 \times 10^{-5}$ (data not shown). While dynamics in such a dilute regime does not show any significant dependence on volume fraction, we find that, as expected, $t$ scales linearly with volume fraction, $t=1.65 \phi$. A conservative estimate of the maximum volume fraction $\phi_{\max }$ for which multiple scattering effects can be safely neglected is obtained as the volume fraction where the transmitted intensity is attenuated by $1 \%$. In this study, this attenuation value is found at $\phi_{\max }=0.5 \%$. 
Our results show that p-DDM allows an effective determination of the roto-translational diffusion of spherical, optically anisotropic particles. It is interesting to compare our approach with recently proposed Digital Fourier Microscopy methods, used for similar purposes [17-19]. Coherent laser light illumination was used both in the seminal work in Ref. [17] and more recently in Ref. [19]. In both cases, a near field scattering setup with optical polarizers was employed successfully to characterize rod-like particles [17] and spherical particles with an optically anisotropic core [19]. While the analysis in Ref. [19] was based on calculating the structure function similarly to what is done in the present work, in Ref. [17] the dynamics was too fast to be captured with a pixel-based detector and a time-exposure scheme was adopted. In both studies, the dynamics of the particles was successfully assessed at the expense of building a specialized optical setup, which might restrict the use of such methods to those laboratories where an optics expertise is available. By contrast, our p-DDM approach is based on a commercial microscope and no prior knowledge of advanced optics is needed for their use. Another difference between p-DDM and near field scattering is that in the latter a laser light source is employed while in the former, sample illumination is obtained with a halogen lamp, whose degree of spatial and temporal coherence can be adjusted with diapraghms and filters. Reducing the light source coherence might help with longer acquisitions, in which laser-based setups usually exhibit tedious time-dependent interference fringes that can impair the success of the measurement [23]. In Ref. [18] the authors have shown that traditional DDM can be used to recover the rotational diffusion coefficient of single optically anisotropic Janus particles but, owing to the peculiar nature of the sample, the study was performed without optical polarizers. This was made possible by the use of large particles (above the diffraction limit) made of two different materials, which become visible because of differential absorption. This approach cannot be easily translated to particles that are arbitrarily small or whose optical anisotropy is not based on absorption. Also, the authors in Ref. [18] treat the translational motion as a disturbance that can cause difficulties in data analysis. Our approach overcomes both difficulties, along the lines that were also suggested in Ref.[18]. Finally, p-DDM could be adapted to be used with particles whose optical asymmetry is more complex than uniaxial [2] and also with particles exhibiting shape anisotropy, such as for instance bacteria. In the latter case, however, the signal would be in general smaller than the one originated by particles with a strong optical anisotropy such as the ones used in this work.

\section{Conclusions}

We have demonstrated polarized differential dynamic microscopy, a method based on a commercial microscope that allows the simultaneous characterization of the translational and rotational diffusion coefficients of optically anisotropic particles. Compared to particle tracking, p-DDM is less subject to artifacts arising from the image processing but at the same time is less effective in obtaining information at the single particle level. With respect to DDLS, p-DDM is currently less sensitive to small colloidal particles, whose scattering cross section scales in the Rayleigh regime as $R^{6}$ [21], but we expect that such gap will be reduced as more sensitive pixel detectors are made available. On the other hand, we envision applications in which p-DDM may even result more suitable than DDLS. For instance, it has been shown that the rotational motion of seed particles in laminar or turbulent flows can be used as a probe of the local fluid vorticity [35]. In this respect, DDLS can easily access the rotational dynamics of an ensemble of small particles but in most cases of interest the shear gradients occurr over a distance that is too large compared with the particle size. If the size of the particles is increased, the roto-translational relaxation rate

$\Gamma_{1}(q)=D_{T} q^{2}+6 D_{R}=D_{T} q^{2}\left[1+\frac{9}{2} \frac{1}{(q R)^{2}}\right]$, is mainly set by translational diffusion unless the second 
term on the r.h.s. becomes dominant. In practice, once the particle radius $R$ is chosen, this implies that one needs to probe the dynamics at small enough $q$ (such that $q R<1$ ), a challenging feat for DDLS experiments. This is not the case for p-DDM that naturally probes the dynamics of large particles for small $q$,with the added bonus of its space-resolving capability. For all these reasons, we envision that p-DDM will be a valid complement rather than a replacement for both DDLS and particle tracking.

\section{Acknowledgments}

We thank Stefano Crotti and Manuel Escobedo-Sánchez for useful discussions and for early contributions to the project. We also thank Roberto Piazza for illuminating discussions. Financial Support for this work was provided by Ministero dell'Istruzione, dell'Università e della Ricerca (MIUR) under the program "Futuro in Ricerca" - Project ANISOFT (RBFR125H0M) and CONACYT-Mexico (Project 166645).

\section{Appendix A. Derivation of Eq. 15}

Aim of this Appendix is deriving Eq. 15, starting from the definition $F_{i}(\mathbf{q}, \Delta t)=\left\langle\hat{s}^{*}(q, 0) \hat{s}(q, \Delta t)\right\rangle$ [15] and from Eq. 14. Under quite general assumptions on the symmetry of the optical system, $\tilde{T}_{A F}\left(\mathbf{q}, q_{z}\right)$ is an even function of both $\mathbf{q}$ and $q_{z}$, while $\tilde{T}_{P F}$ is an even function of $\mathbf{q}$ and an odd function of $q_{z}[14]$. We can make use of these symmetries to obtain

$$
\begin{aligned}
F_{i}(\mathbf{q}, \Delta t) & =\int d q_{z}\left|\tilde{T}_{A F}\left(\mathbf{q}, q_{z}\right)\right|^{2} F_{A A}\left(\mathbf{q}, q_{z}, \Delta t\right)+ \\
& +\int d q_{z}\left|\tilde{T}_{P F}\left(\mathbf{q}, q_{z}\right)\right|^{2} F_{P P}\left(\mathbf{q}, q_{z}, \Delta t\right)
\end{aligned}
$$

where we have introduced the (non-normalized) intermediate scattering functions $F_{A}\left(\mathbf{q}, q_{z}, \Delta t\right)=\left\langle\tilde{c}_{A}^{*}\left(\mathbf{q}, q_{z}, 0\right) \tilde{c}_{A}\left(\mathbf{q}, q_{z}, t\right)\right\rangle$ and $F_{P}\left(\mathbf{q}, q_{z}, \Delta t\right)=\left\langle\tilde{c}_{P}^{*}\left(\mathbf{q}, q_{z}, 0\right) \tilde{c}_{P}\left(\mathbf{q}, q_{z}, t\right)\right\rangle$ for amplitude and phase, respectively. Using Eqs. 5, 6, 11 and 10, we can rewrite Eq. A.1 as a function of the particles positions and orientations. If the particles are spherical, as in the case of our experiment, even in presence of non-negligible inter-particle interactions, the orientations of the optical axes of different particles are uncorrelated [2] and one gets

$$
\left\langle Y_{2, m}^{*}\left(\theta_{n_{1}}(0), \phi_{n_{1}}(0)\right) Y_{2, m^{\prime}}\left(\theta_{n_{2}}(\Delta t), \phi_{n_{2}}(\Delta t)\right)\right\rangle=\delta_{n_{1}, n_{2}} \delta_{m, m^{\prime}} \frac{1}{4 \pi} F_{R}(\Delta t)
$$

where $F_{R}(t)$ is a rotational correlation function [4]. The spatial correlation properties of the sample can be described in terms of the collective and self- dynamic structure factors, which are defined as:

$$
\begin{aligned}
F_{S}\left(\mathbf{q}, q_{z}, \Delta t\right)= & \frac{1}{N} \sum_{n}\left\langle e^{-j \mathbf{q} \cdot\left[\mathbf{x}_{n}(\Delta t)-\mathbf{x}_{n}(0)\right]} e^{-j q_{z}\left[z_{n}(\Delta t)-z_{n}(0)\right]}\right\rangle \\
F_{C}\left(\mathbf{q}, q_{z}, \Delta t\right) & =\left\langle\hat{c}\left(\mathbf{q}, q_{z}, 0\right) \hat{c}^{*}\left(\mathbf{q}, q_{z}, \Delta t\right)\right\rangle= \\
& =\frac{1}{N} \sum_{n_{1}, n_{2}}\left\langle e^{-j \mathbf{q} \cdot\left[\mathbf{x}_{n_{1}}(\Delta t)-\mathbf{x}_{n_{2}}(0)\right]} e^{-j q_{z}\left[z_{n_{1}}(\Delta t)-z_{n_{2}}(0)\right]}\right\rangle
\end{aligned}
$$

Making use of the these definitions, we can recast Eq. A.1 in the form

$$
\begin{aligned}
F_{i}(\mathbf{q}, \Delta t) & =\int d q_{z} A_{1}\left(\mathbf{q}, q_{z}\right) P\left(\mathbf{q}, q_{z}\right) F_{C}\left(\mathbf{q}, q_{z}, \Delta t\right)+ \\
& +\int d q_{z} A_{2}\left(\mathbf{q}, q_{z}\right) P\left(\mathbf{q}, q_{z}\right) F_{S}\left(\mathbf{q}, q_{z} \Delta t\right) F_{R}(\Delta t)
\end{aligned}
$$


where we have explicitly introduced the form factor $P\left(\mathbf{q}, q_{z}\right)$ of the particles and where $A_{1}$ and $A_{2}$ are two new effective transfer functions associated to the collective translational diffusion and to the self- roto-translational diffusion, respectively:

$$
\begin{aligned}
& A_{1}=\left(k^{3} \alpha\right)^{2}\left|T_{P}\right|^{2}+\left(\frac{2}{3} k^{6} \alpha^{2}+\frac{4}{27} k^{6} \beta^{2}\right)^{2}\left|T_{A}\right|^{2} \\
& A_{2}=\frac{4}{45}\left(k^{3} \beta\right)^{2}\left(1+\frac{3}{4} \tan ^{2} \gamma\right)\left|T_{P}\right|^{2}+\frac{16}{3645}\left[k^{6}\left(\alpha_{3}^{2}-\alpha_{1}^{2}\right)\right]^{2}\left(1+\frac{27}{16} \tan ^{2} \gamma\right)\left|T_{A}\right|^{2}(\text { A. } 7)
\end{aligned}
$$

A relatively simple expression for $A_{1}$ and $A_{2}$ can be obtained for the case of a dilute suspension of non-interacting particles. In this case $F_{R}(t)=e^{-6 D_{R} t}$, where the $D_{R}$ is the Stokes-Einstein rotational diffusion coefficient and $F_{C}(\mathbf{q}, t)=F_{S}(\mathbf{q}, t)=e^{-D_{T} q^{2} \Delta t}$, where $D_{T}$ is the Stokes-Einstein rotational diffusion coefficient. Moreover, as discussed in [14, 24] for Brownian particles, the axial dynamics does not substantially affect the image correlation functions. In the low- $q$ regime, Eq. A.5 can be approximated with its $2 D$ form:

$$
F_{i}(\mathbf{q}, \Delta t) \simeq P(\mathbf{q})\left[A_{1}^{\prime}(\mathbf{q}) e^{-D_{T} q^{2} \Delta t}+A_{2}^{\prime}(\mathbf{q}) e^{-\left(D_{T} q^{2}+6 D_{R}\right) \Delta t}\right]
$$

where $A_{1 / 2}^{\prime}(q)=\int d q_{z} A_{1 / 2}\left(\mathbf{q}, q_{z}\right)$.

\section{References}

[1] Berti D and Palazzo G 2014 Colloidal Foundations of Nanoscience (Newnes)

[2] Berne B J and Pecora R 2000 Dynamic light scattering: with applications to chemistry, biology, and physics (Dover Publications)

[3] Borsali R and Pecora R 2008 Soft Matter Characterization (Springer)

[4] Piazza R, Degiorgio V, Corti M and Stavans J 1990 Phys. Rev. B 424885

[5] Degiorgio V, Bellini T, Piazza R and Mantegazza F 1997 Phys. A 235 279-290

[6] Waigh T A 2005 Rep. Prog. Phys 68685

[7] Cicuta P and Donald A M 2007 Soft Matter 3 1449-1455

[8] Piazza R 1993 Phys. Scripta T49A 94-98

[9] Jabbari-Farouji S, Eiser E, Wegdam G H and Bonn D 2004 J. Phys. Condens. Matter 16 L471

[10] Mériguet G, Dubois E, Dupuis V and Perzynski R 2006 J. Phys. Condens. Matter 1810119

[11] Edmond K V, Elsesser M T, Hunter G L, Pine D J and Weeks E R 2012 Proc. Natl. Acad. Sci. U.S.A. 109 17891-17896

[12] Dehaoui A, Issenmann B and Caupin F 2015 Proc. Natl. Acad. Sci. U.S.A. 112 12020-12025

[13] Cerbino R and Trappe V 2008 Phys. Rev. Lett. 100188102

[14] Giavazzi F, Brogioli D, Trappe V, Bellini T and Cerbino R 2009 Phys. Rev. E 80031403

[15] Giavazzi F and Cerbino R 2014 Journal of Optics 16083001

[16] Giavazzi F, Crotti S, Speciale A, Serra F, Zanchetta G, Trappe V, Buscaglia M, Bellini T and Cerbino R 2014 Soft matter 10 3938-3949 
[17] Brogioli D, Salerno D, Cassina V, Sacanna S, Philipse A P, Croccolo F and Mantegazza F 2009 Optics express $\mathbf{1 7} 1222-1233$

[18] Wittmeier A, Leeth Holterhoff A, Johnson J and Gibbs J G 2015 Langmuir 31 10402-10410

[19] Escobedo-Sánchez M A, Hugo A, Arauz-Lara J L, Haro-Pérez C and Rojas-Ochoa L F 2015 J. Chem. Phys. 143044902

[20] Degiorgio V, Potenza M and Giglio M 2009 Eur. Phys. J. E Soft Matter 29 379-382

[21] Van De Hulst H 1957 Light scattering by small particles (Wiley)

[22] Potenza M, Sanvito T, Alaimo M, Degiorgio V and Giglio M 2010 Eur. Phys. J. E Soft Matter 31 69-72

[23] Cerbino R and Vailati A 2009 Curr. Opin. Colloid Interface Sci. 14 416-425

[24] Lu P J, Giavazzi F, Angelini T E, Zaccarelli E, Jargstorff F, Schofield A B, Wilking J N, Romanowsky M B, Weitz D A and Cerbino R 2012 Phys. Rev. Lett. 108

[25] Mertelj A, Arauz-Lara J L, Maret G, Gisler T and Stark H 2002 Europhys. Lett. 59337

[26] Díaz-Leyva P, Pérez E and Arauz-Lara J L 2004 J. Chem. Phys. 121 9103-9110

[27] Haro-Pérez C, Andablo-Reyes E, Díaz-Leyva P and Arauz-Lara J L 2007 Phys. Rev. E 75 041505

[28] Bibette J 1991 J. Colloid Interface Sci. 147 474-478

[29] Wilson L G, Martinez V A, Schwarz-Linek J, Tailleur J, Bryant G, Pusey P and Poon W C 2011 Physical review letters 106018101

[30] Ferri F, D'Angelo A, Lee M, Lotti A, Pigazzini M C, Singh K and Cerbino R 2011 Eur. Phys. J.-Spec. Top. 199 139-148

[31] He K, Spannuth M, Conrad J C and Krishnamoorti R 2012 Soft Matter 8 11933-11938

[32] Germain D, Leocmach M and Gibaud T 2015 arXiv preprint arXiv:1511.00923

[33] Safari M S, Vorontsova M A, Poling-Skutvik R, Vekilov P G and Conrad J C 2015 Phys. Rev. E 92042712

[34] Mailer A G, Clegg P S and Pusey P N 2015 J. Phys. Condens. Matter 27145102

[35] Tong P, Xia K Q and Ackerson B 1993 J. Chem. Phys. 98 9256-9265 